EPJ Web of Conferences 114,02100 (2016)

DOI: 10.1051 /epjconf/201611402100

C) Owned by the authors, published by EDP Sciences. 2016

\title{
Hybrid solar receiver as a source of high-temperature medium for an absorption chiller supply
}

\author{
Estera Przenzak ${ }^{1, a}$ and Mariusz Filipowicz ${ }^{1}$ \\ ${ }^{1}$ AGH University of Science and Technology, Faculty of Energy and Fuels, Department of Sustainable Energy Development, Mickiewicza \\ Av. 30, 30-059 Krakow, Poland
}

\begin{abstract}
This article discusses the problems related with the cold production, i.e. energy efficiency of the process. The idea of solar cooling systems has been presented as the solution of the problem of big electricity demand. The paper discusses the principle of the operation of absorption chillers. Disadvantages and advantages of the solar cooling systems were discussed. The installation for manufacturing high-temperature heat based on solar collectors and concentrator of solar radiation constructed in AGH in Cracow has been presented. This installation is a first stage of projected, complete solar cooling system. The special attention is paid to the dedicated solar high-temperature heat receiver as a most important element of the system. The achieved values of temperature, power and efficiency depending on the working medium flow has been presented and discussed. The intensity of solar radiation during the measurements has been taken into account. Two versions of heat receiver were investigated: non-insulated and insulated with mineral wool. The obtained efficiency of the heat receiver (less than $30 \%$ ) is not satisfactory but possibility of improvements exist.
\end{abstract}

\section{Introduction}

In the developed countries, the clear increase in energy demand for refrigeration and air conditioning has been observed for many years. The reason for this is the general desire of the society to increase the thermal comfort throughout the year. The increasing energy consumption contributed to the formation of the currently ongoing boom to promote alternative energy sources. At the same time the steps are undertaken aimed to reduce the demand for electricity by, among others, modernising the lighting, using modern energy-efficient household and electronic devices or thermo-modernisation of buildings. An additional factor influencing such changes includes the European and global restrictions announced in Directives and legal regulations [1-3].

The production of cool requires a lot of electricity. This applies especially to the summer period, when cool is used not only in catering or medicine, but also in air conditioning. The study [4] presents the analysis of the energy demand in residential buildings with a different shape coefficient. The authors analysed, among others, the energy use for heating and cooling purposes during the year on the example of Canada (figure 1). The works has been performed based on computer simulations using the eQuest software. Calculations were performed for buildings with a different orientation of elevation relative to world sides and with varied proportions of their length. Profiles visible in chart 1 mean:
- G90 ${ }^{\circ} \Theta$ profile: a building oriented with shorter walls to north/south with wall proportions 1:4.2;

- profile C: a building oriented with longer walls to north/south with wall proportions 1.5:1.

In figure 1 can be seen that regardless of the geometry of the building the greater demand for energy needed to maintain thermal comfort occurs in the winter period.

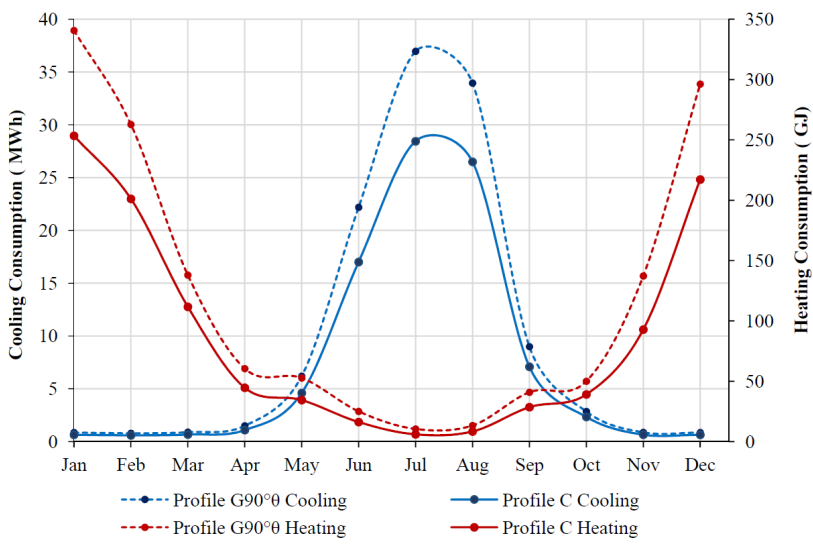

Figure 1. Comparison of energy consumption between two building profiles with different aspect ratio and orientation for Toronto simulations [4].

However, for the purpose of supplying the central heating installation the solid or gas (less often oil fuel boilers are used most commonly. Thus, the demand for

\footnotetext{
${ }^{\mathrm{a}}$ Corresponding author: esterap@agh.edu.pl
} 
electricity in the winter period is not as high. While in order to improve the quality of indoor air in the summer period most often the compressor air conditioners are used, which are powered by electricity. Hence the emerging problem of high electricity consumption during hot weather. According to [5] the demand for electricity needed to maintain the thermal comfort conditions in the summer period is much higher than in the winter period.

In the European Union, $55.1 \%$ of electricity comes from coal thermal power plants, $28 \%$ from nuclear power plants, while less than $17 \%$ from renewable energy sources of various kinds [6]. The biggest share in electricity production, not only in Europe but also around the world, comes from carbon power plants. They are characterised by high inertia, therefore energy safety during summer is at risk. The demand for electricity is not evenly distributed in time. Difficult to predict weather conditions are sometimes rapid within days, and sometimes even hours. This means that during the period of strong heat, users suddenly overload the power grid. Due to the inertia of power plants, energy suppliers cannot always ensure the continuous supply of electricity. Dangerous situations of overloads an thus shut-offs of networks because of the too high consumption of energy needed to power air conditioning devices happened, among others, in China [7] or the United States [8]. Also the effects associated with strong heat were observed in Poland in August 2015, when the amount of generated power dropped in the power system due to water shortages (and its too high temperature) for cooling power units. In addition, high electricity consumption (derived from conventional sources) for the purposes of producing cold causes the increased emission of carbon dioxide to the atmosphere [9] which in the long term has a negative impact on the environment.

Comparing the blue curve (online version) in figure 1 with the graph of the average global intensity of solar radiation within a year (figure 2) it can be seen that these distributions are similar. Data presented in graph 2 were created in order to assess different methods of modelling of weather conditions presented in [10].

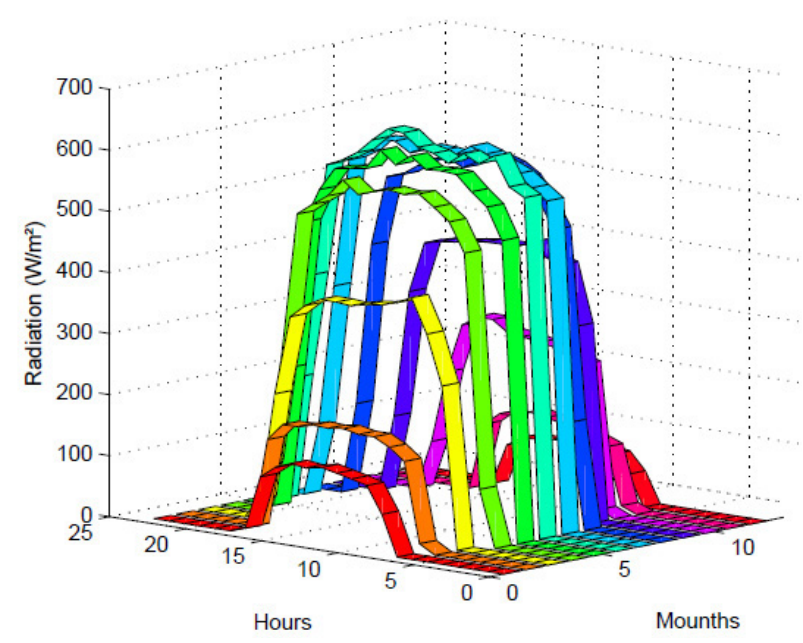

Figure 2. Measured monthly average daily global radiation [10].
Measurements of solar radiation were performed in northern France (Méteo France ground station of the Troyes-Barbery), i.e. in a place characterised by a relatively high cloudiness throughout the year. The presented coherence is the basis for research on modern cooling systems based on solar energy. It is possible by applying the sorption refrigerator, i.e., a heat-powered device.

\subsection{Fundamentals of sorption refrigeration}

In sorption aggregates the working medium is the twocomponent medium, i.e., the solution of sorbent and substances absorbed by it. In sorption chillers the absorber is in the solid form, and the adsorption process takes place at the phase boundary. In case of absorption chillers the liquid absorbent is used, so the sorption process takes place in the whole volume of the medium. Most often two types of absorption chillers are used: bromine-lithium $\left(\mathrm{LiBr}-\mathrm{H}_{2} \mathrm{O}\right)$ and ammonia $\left(\mathrm{H}_{2} \mathrm{O}-\mathrm{NH}_{3}\right)$. In the first ones, water plays the role of the refrigerant, so they are used in systems, where chilled water has the temperature not smaller than approx. $5^{\circ} \mathrm{C}$. While the ammonia absorption chillers can be used to obtain lower temperatures (even up to $-60^{\circ} \mathrm{C}$ ), however they require higher investment outlay. Compared to conventional compressor devices, absorption chillers are characterised by lower noise level, lower service costs (a small number of moving parts) and a much longer period of exploitation, because even to 25-20 years (for the compressor refrigerator it is approx. 15 years) [11].

As in the compressor refrigerators, also in the absorption devices in the evaporator the absorption of heat takes place by the medium with low pressure, while in the condenser at high pressure this heat is released. The change of pressure takes place by using a throttling valve and the absorption-desorption system, replacing the compressor (figure 3).

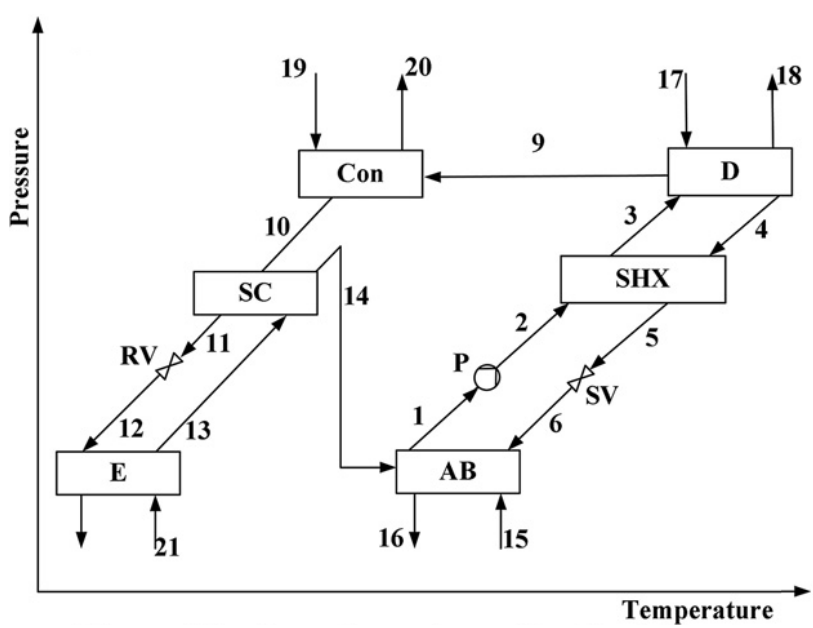

Figure 3. An exemplary scheme of the absorption refrigerator [12]: AB-absorber, P-solution pump, SHX-solution heat exchanger, SV-solution expansion valve, D-desorber, Concondenser, SC-refrigerant subcooler, RV- refrigerant expansion valve, E-evaporator. 
The refrigerant of low pressure and in the liquid form, flowing through the evaporator, takes the heat from the ice water led to it, thus reducing its temperature. Ice water can be then used to distribute cold in the rooms, e.g., using the fan coil unit. Vapour of the refrigerant created in the absorber flow to the system with the absorber, where in contact with the absorbing medium they are subject to absorption. In this process the dissolution heat is released, which must be discharged from the system using the external system with the cooling water. The rich solution created in the absorber is pumped using a circulation pump to the desorber. In this place the high-temperature heat is applied, which is usually generated using gas or electric heater. Increasingly, it is also a waste heat created as a result of other technological processes or heat produced using solar installations. In the desorber, using the hightemperature heat led to the system, the cooling medium is evaporated from the absorbent. The absorbent (weak solution) flows back to the absorber. In contrast, the refrigerant vapour goes to the condenser, where by means of cooling water from the external system flowing through the exchanger, they are condensed. The liquid working medium created this way is directed through the throttling valve in order to achieve low pressure, required to conduct the evaporation process in the evaporator.

\subsection{Review of solar sorption cooling systems}

Studies on such systems are carried out around the world. For example, in Poland at the Department of Engineering and Environmental Protection of the Czestochowa Technical University studies on the possibility to create conditions of thermal comfort in summer in the room with the capacity of $30 \mathrm{~m}^{3}$ were conducted. The research work was carried out based on adsorption refrigerator powered by heat produced by the installation of five flat solar collectors. It has been proven that it is possible to use the standard installation with solar collectors for the purposes of cold production. However, we should bear in mind that the thermal effectiveness coefficient TEC is in this case quite low and is approx. 0.6-0.7 [13, 14]. This is due to the fact that solar collectors only produce heat with low temperature (approx. $60^{\circ} \mathrm{C}$ ). Compared to absorption aggregates, adsorption ones are characterised by a much lower thermal effectiveness coefficient. Nevertheless, studies on solar power supplies of this type of refrigeration are conducted around the world on a large scale. The overview of modern solutions and description of future trends in solar adsorption installations was described, among others in $[15,16]$.

At the same time in the energy industry we observe the dynamic development of solar absorption systems. In Oberhausen in Germany in summer months the system with the absorption cooling aggregate with the power of $34 \mathrm{~kW}$ has been tested, powered by vacuum solar collectors with the surface of $108 \mathrm{~m}^{2}$. To facilitate the operation of the system, it was equipped with the cooling tower with the capacity of $134 \mathrm{~kW}$. As a result, over the 5 -year exploitation of the installation $25 \%$ cold was produced needed to meet the demand for rooms with a total area of $270 \mathrm{~m}^{2}$. At this time, the TEC coefficient values of the used cooling device were quite low and amounted to $0.37-0.81$ [17].

Similar studies were carried out in Madrid, where the TEC coefficient of the bromine-lithium absorption chiller with the power of $35 \mathrm{~kW}$ was $0.23-0.42$. This system was also powered only by the installation with solar (flat) collectors. The cooling aggregate in this case achieved the maximum of only $7.5 \mathrm{~kW}$ power ( $21 \%$ nominal) [18].

For sorption devices, and particularly absorption refrigerators, to work with high efficiency, it is necessary to provide desorption heat to the system with temperature over $85^{\circ} \mathrm{C}$. This is shown in the graph (figure 4 ) of the dependency of cooling efficiency of the absorption refrigerator on the nominal power of $17.6 \mathrm{~kW}$ on temperature of the medium flowing into the desorber. It is an example of energy characteristics included in the catalogue card of the YAZAKI aggregate [19].

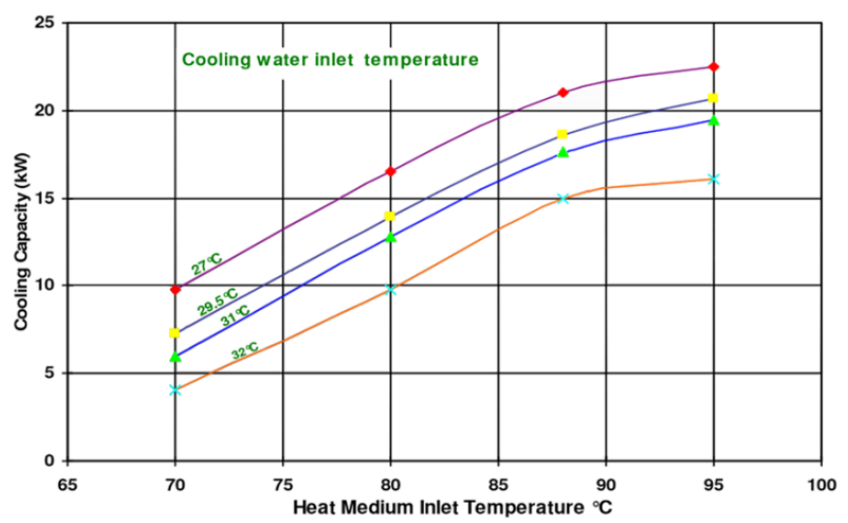

Figure 4. Cooling capacity of the absorption device depending on temperature of the heating medium [19].

From the graph shown in figure 4 it can be seen that for the absorption systems to work efficiently it is necessary to provide the high-temperature heat to the system. Therefore, in solar systems used for generating cold, the systems concentrating the solar radiation have a great potential. The concentrated light can in fact be used to increase the temperature of the working medium preheated in collectors, or be used as the only source of heat in this system.

Studies on focus systems used to produce cold were conducted, among others, in Seville in Spain, where the system focusing light in the using linear Fresnel lens with the total surface of $352 \mathrm{~m}^{2}$ has been constructed. In the discussed studies the two-stage bromine-lithium cooler was used with the power of $174 \mathrm{~kW}$ as well as the system for heating the working medium in the gas burners. The conducted measurements showed that in the period from May to October, i.e., when there is the largest demand for cold, the cooler works with the TEC effectiveness at the level of 1.1-1.25. Furthermore, the SHF factors (Solar Heat Fraction - the ratio of thermal energy to the one obtained from solar installation to thermal energy powering the desorber) and SCR (Solar Cooling Ratio the efficiency of the whole system) are at a very high level, respectively $75 \%$ and $44 \%$. In the discussion of research works also the problems, which concern the 
focus systems, were indicated. Because it has been studied that on cloudy days (predominance of scattered radiation) the use of gas burners in the discussed system is necessary and constitutes the production of approx. $60 \%$ energy needed for the appropriate increase of temperature of the working medium. Also the issue of the impact of the focus installation pollution on the effectiveness of the whole system has been raised (decrease of efficiency even by 50\%) [20].

While in Tunisia at the Centre of Energy Research and Technologies in Bordj-Cedria the system of trough solar radiation concentrators has been constructed and tested, which powers the absorption cooler (BrLi) with the nominal power of $16 \mathrm{~kW}$, which in turn was used as powering for several fan coils installed in individual rooms of the building. In this case the device used to heat the working medium was also used (electrical heater). In order to increase the SHF factor the buffer tank was used, which was applied for storing heat to be used at night. The solar degree of coverage thanks to this operation increased from the value of 0.54 up to 0.77 . In the discussed system, the cooler reached the maximum power at the level of $75 \%$ of its nominal value, i.e., the maximum of $12 \mathrm{~kW}$ has been achieved. On the basis of a number of conducted measurements and calculations it has been shown that the TEC factor of the studied absorption aggregate is in the range of 0.8-0.91 [21].

Analysing the discussed solutions it can be seen that the coefficient of thermal effectiveness in the case of applying the system focusing the solar radiation is higher than in systems powered by solar collectors. The review of systems using the solar radiation energy for powering sorption systems has been performed, among others, in $[11,22-24]$

\section{Solar system for high-temperature heat generation}

At the Department of Sustainable Energy Development in the Faculty of Energy and Fuels at AGH in Cracow there are ongoing works on the construction of the solar cooling system analogous to the described ones. The target system consists of the hybrid connection of solar collectors with the parabolic concentrator of solar radiation. The system will also use the absorption aggregate, which has not yet been mounted.

Figure 5 presents the diagram of the proposed solution. According to the idea of the described system, the working medium (thermal oil) is pumped to solar collectors (1) by using the circulation pump (7). Then, the pre-heated medium goes to the heat exchanger (3) located near the focal point of the concentrator, where its temperature increases. The resulting heat is used to power the absorption cooler (4), which in turn supplies chilled water to the fan coil units. The remaining amount of heat is accumulated in the buffer tank (6) and is used for heating the utility water. The expansion vessel (8) (just like in classic installations) protects the installation against the excessive pressure increase. Given that this is the high-temperature installation, the expansion vessel was additionally equipped with the cooling vessel. On colder days (spring, autumn), when air conditioning devices are not used, the heated medium is directed by the three-way valves directly to the coil of the buffer tank. When the accumulation tank has the already sufficient amount of the heat, the working medium after leaving the absorption cooler, is directed directly to solar collectors (it omits the tank). The system was equipped with the fan cooler (5), which serves as the protection function in case of achieving too high temperatures at the outlet of the sorption aggregate.

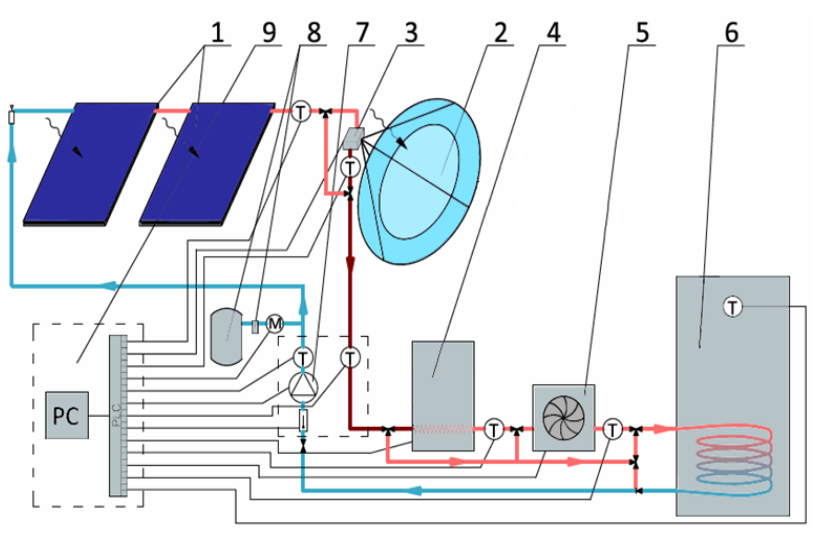

Figure 5. The diagram of solar collector system with the solar radiation concentrator for absorption cooling.

Currently, the system consists of two flat solar collectors mounted on the roof connected with the system of solar radiation concentrator (figure 6a). The concentrator is mounted on the tracker using the differential sensor built of six LED diodes. This type of tracker allows the quite accurate tracking of the sun location, what in case of focus systems has a significant meaning. The concentrator is built of the bowl with a diameter of $1.8 \mathrm{~m}$ lined with the reflective foil and the mirror deposited on it with a diameter of $1.2 \mathrm{~m}$. On the roof there is also a fan cooler. Due to the moving elements of the installation, piping is made of corrugated steel pipes in the rubber coating.

While in the laboratory other installation elements have been mounted, which are visible in figure 6b. Only the absorption aggregate has not yet been installed.

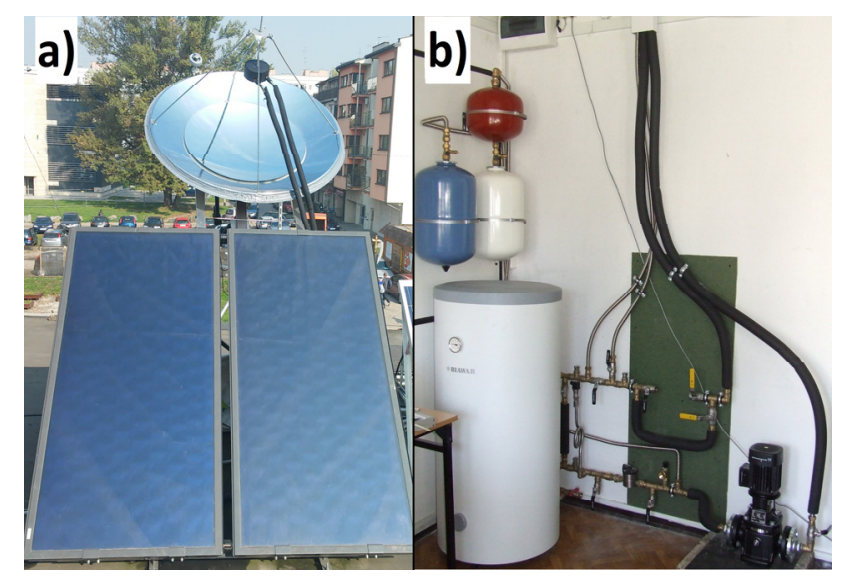

Figure 6. View of the hybrid installation of solar collectors with a concentrator a) on the roof, b) in the laboratory. 
The system was equipped with the system of control and measurement automation based on the PLC controller (figure 7). The following measurement elements were used:

- oil temperature sensors in different points of the system (also outside air temperature) $\left({ }^{\circ} \mathrm{C}\right)$,

- pressure sensor (bar),

- oval-wheel flow meter (1/ $\mathrm{min})$,

- solar radiation intensity sensor $\left(\mathrm{W} / \mathrm{m}^{2}\right)$.

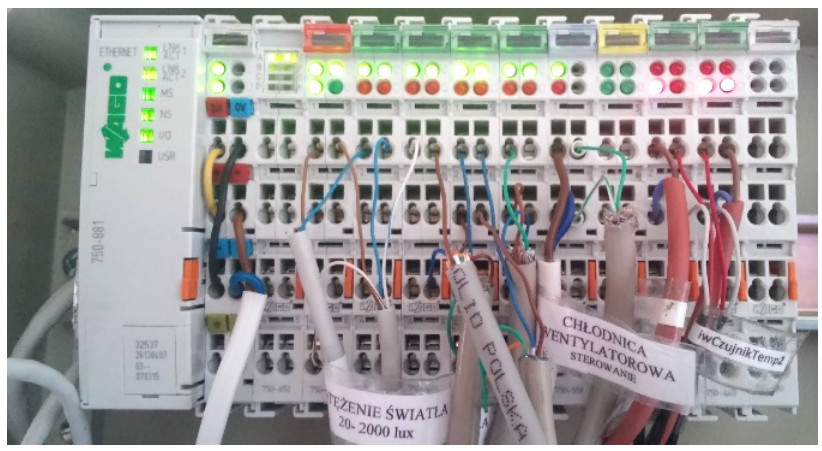

Figure 7. PLC controller of the WAGO company with connected measurement system elements.

Using the industrial programmable controller it is also possible to control the performance of the circulation pump and fan radiator. Preview of data, their recording and control of individual parameters takes place through the visualisation presented in figure 8 performed in the CoDeSys software.

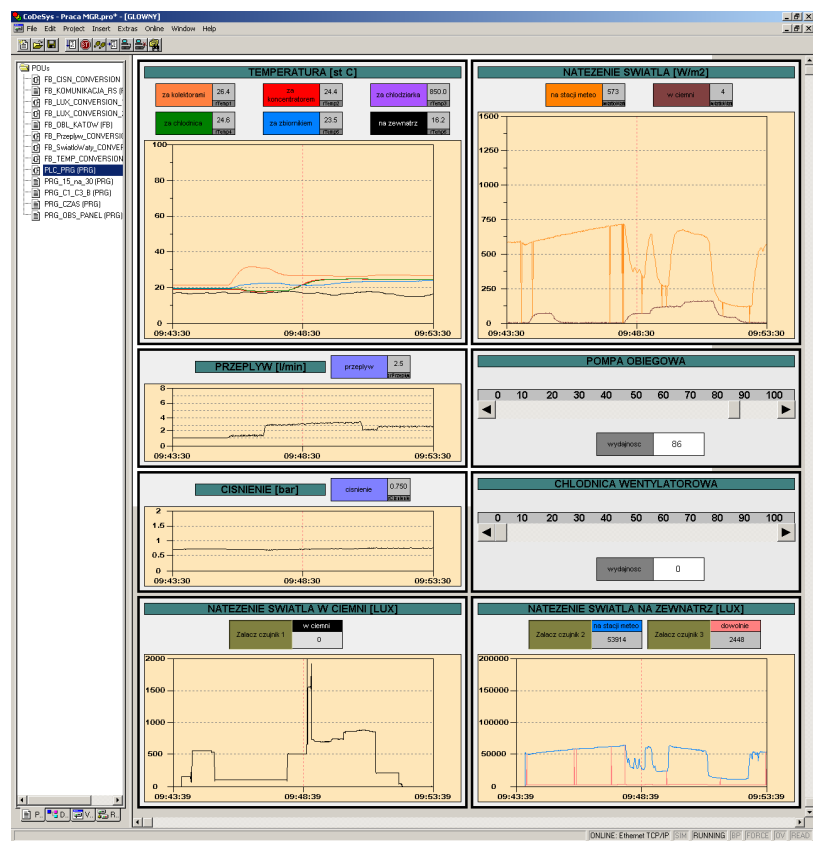

Figure 8. Control and data recording panel.

One of the most important elements of the discussed installation is the receiver of high-temperature heat absorbing the concentrated solar radiation. In the first version, the receiver is made using two interconnected aluminium blocks with the channel milled inside. The diameter of the receiver is $18 \mathrm{~cm}$ (figure 9). The surface, on which the concentrated solar radiation falls was dulled and blackened. Simultaneously to the experimental studies the simulation studies are performed. They serve as an aid in optimising the receiver system, namely: radiation absorption, its conversion and high-temperature heat reception. Effectiveness of the listed processes depends, among others, on the external shape and internal geometry of the receiver, type of absorption surface, receiver location in the concentrator geometrical system, as well as the proper flow values depending on the current parameters of the working medium.
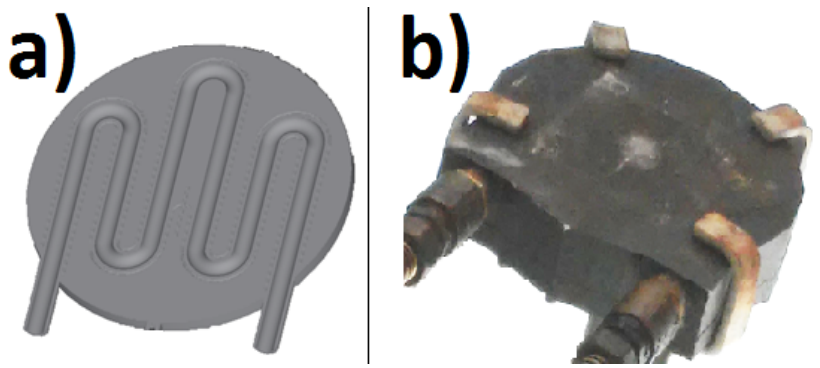

Figure 9. High-temperature heat receiver a) cross section, b) view of the receiver in the mounting holder.

\section{Solar system for high-temperature heat generation}

In summer 2015 studies on the possibility to produce heat using only the solar radiation concentrator were performed. During measurements, the working medium flowed also through the system with two solar collectors, but for the purposes of studies they have been covered. The effectiveness of conversion and heat absorption from the concentrator were tested using the receiver presented in figure 9. Two versions of the receiver were studied: without insulation and with insulation (coating of mineral wool). Insulation of $2 \mathrm{~cm}$ thickness was fastened to the back and lateral surfaces of the receiver. In graphs figure 10 and 11 the change of temperature of the working medium has been presented before and behind the concentrator and with the buffer tank with utility water. The dotted line marks the change in performance of the circulation pump beginning from $100 \%$ to $40 \%$ in case of the insulated receiver. Below the graphs the solar radiation intensity during the study is also presented.

It can be seen that small decreases in solar radiation intensity are caused by the temporary cloudiness, such as, e.g., figure 10: 5 and 26 minute of measurement or figure 11 at: $1 \mathrm{~h} 17 \mathrm{~min}, 1 \mathrm{~h} 36 \mathrm{~min}, 2 \mathrm{~h} 08 \mathrm{~min}$, do not affect the temperature change of the working medium. This results from the inertia of the heat absorption system, which is mainly caused by the volume of oil in the receiver and its heat capacity.

Despite shadowing of solar collectors to some extent they contribute to the heating of medium in the installation. Therefore, the oil temperature before the solar radiation concentrator is higher than its temperature behind the tank. Only when the pump capacity is at the level of $50-55 \%$ in the measurement using the insulation on the receiver the lower temperature was noted on the way between the tank and the concentrator. This is caused by the heat losses in the pipes. 


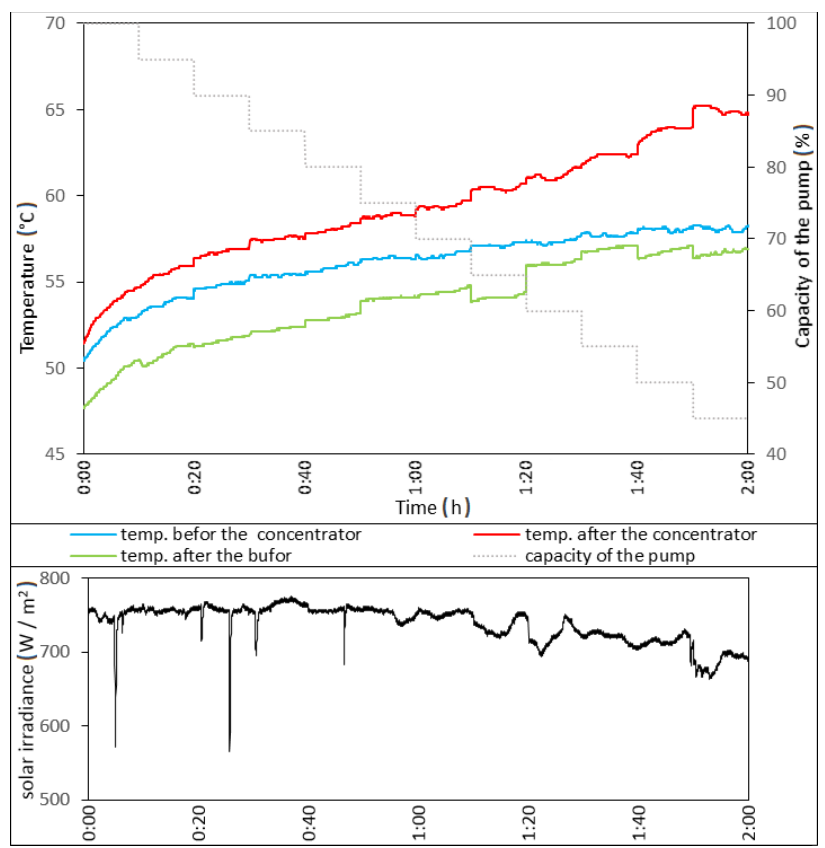

Figure 10. Medium temperature variation for the non-insulated receiver.

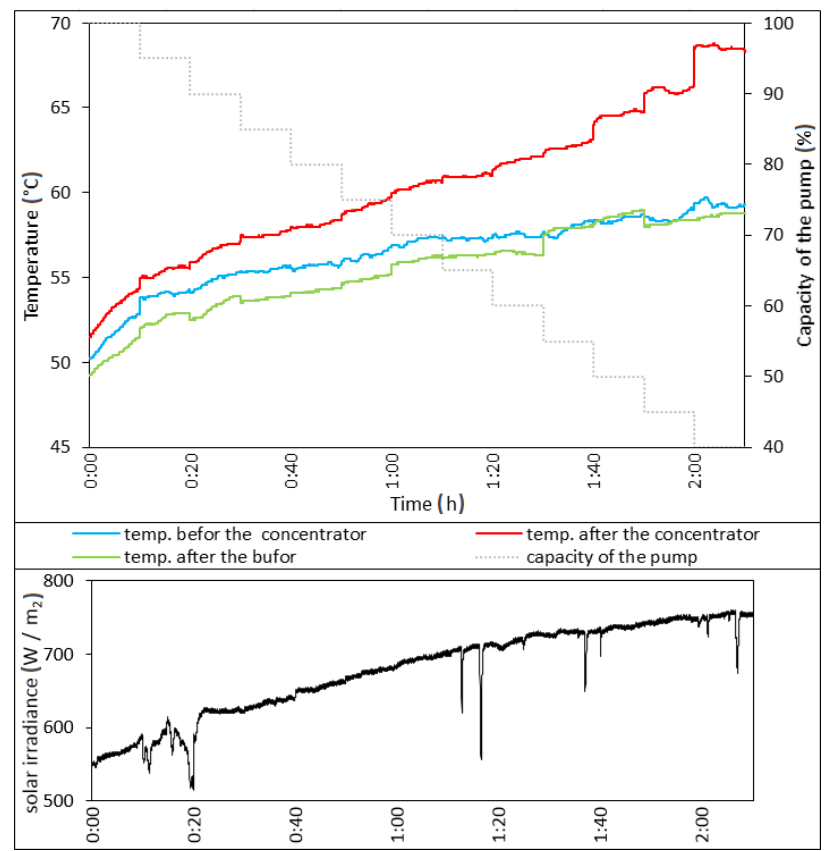

Figure 11. Medium temperature variation for the insulated receiver.

In graph in figure 10 we can see that the decrease of the flow is due to the change of pump capacity from $70 \%$ to $65 \%$ (time moment $1: 10 \mathrm{~h}$ ) caused a slightly more effective heat exchange in the buffer tank (stated based on medium temperature observation in the buffer tank). It is likely that this has caused high enough temperature increase in the tank that the temperature difference between the working medium and the utility water dropped. Therefore, in both cases (non-insulated and insulated receiver) the adjustment of pump capacity under $60 \%$ caused the temperature increase behind the tank - what means the worse heat exchange in the buffer in relation to other capacities. The presented graphs also show that significant temperature changes are mostly observed during the work of a pump with the capacity of $40 \%-80 \%$. The largest increase of oil temperature behind the receiver was observed with the lowest flows. This particularly applies to the insulated tank, where the temperature increases are very clear with the pump capacity from $55 \%$ to $40 \%$. Due to the risk of overheating, the pump cannot work with the yield lower than minimal, which is defined as a certain part of nominal performance in relation to liquid temperature in the discussed case this is $1,5 \mathrm{~L} / \mathrm{min}$ in temperature range to $80^{\circ} \mathrm{C}$. Therefore, measurements were limited to the minimal efficiency of $40 \%$ which gives approx. $1,8 \mathrm{~L} / \mathrm{min}$.

Due to the fact that the measurements were started from the maximum flow rate, which was then reduced during studies, graphs in figure 12-14 present results in reference to the decreasing scale of its value. In order to compare the operation of the receiver working without insulation and with insulation, a graph of the average temperature growth depending on the average flow was made (figure 12). The arithmetic mean values were calculated from 10-minute time intervals during which the fixed value of the circular pump was set. In figure 12 there are also the average values of solar radiation intensity prevailing at the given time of measurement.

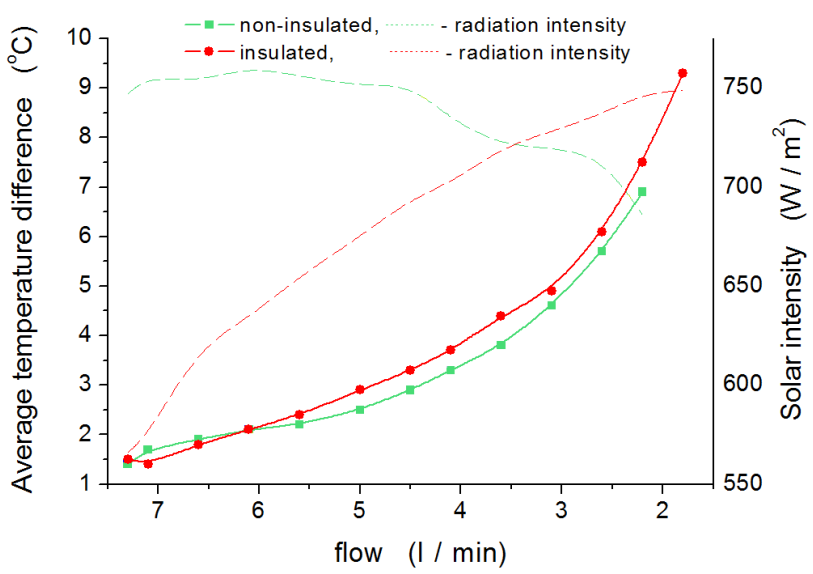

Figure 12. Comparison of the average temperature increase from the flow in case of the receiver without and with thermal insulation.

It can be seen that the greater increase of temperature was achieved in the case of the insulated receiver. However, it may be noted that the study also had the increase of the solar radiation intensity in contrast to the study marked with green curves (online version) - no insulation. Certainly different light intensity has also had a significant impact on the operation of the installation. This can be especially seen at the flow rate of $7.1 \mathrm{~L} / \mathrm{min}$, where the lower temperature increase is noted for the red curve (online version), while the radiation intensity is approx. $180 \mathrm{~W} / \mathrm{m}^{2}$ lower than in case of the study marked with the green curve (online version). Only when this difference reaches approx. $100 \mathrm{~W} / \mathrm{m}^{2}$ (with the flow rate of $5.6 \mathrm{~L} / \mathrm{min}$ ) the use of thermal insulation causes the achievement of higher temperatures. An important information is that despite the drop of the solar radiation 
intensity, we can observe a significant increase of temperature caused by the flow regulation. What is also promising is the fact that in the first version of the heat reception system presented in this article, it was possible to achieve the temperature increase already at the level of over $9{ }^{\circ} \mathrm{C}$.

Knowing the oil temperature before and behind the receiver and the basic properties of oil the power of the receiver was calculated for different flows of the working medium. Results are presented in graph in figure 13.

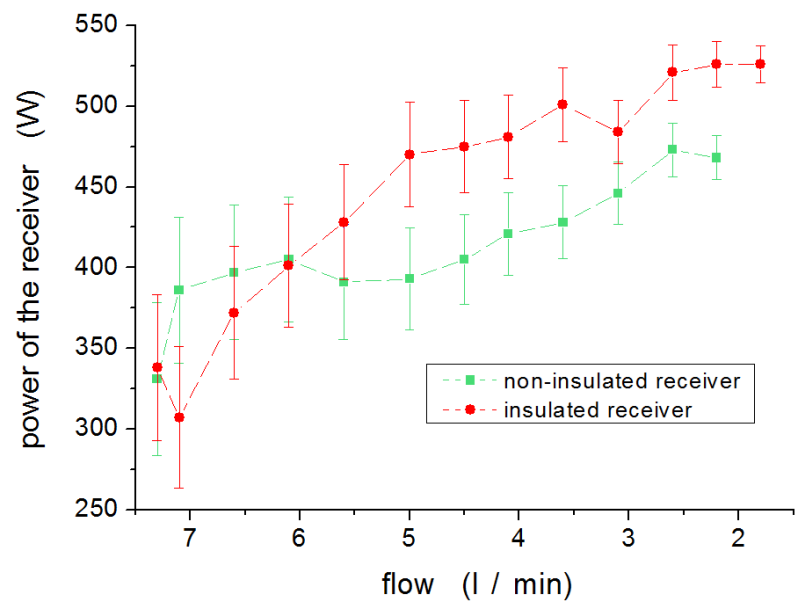

Figure 13. The power of the receiver for varied flow.

In the graph in figure 13 it can be seen that the higher power was obtained in the case of the insulated receiver. In general, the power of the receiver increases with the decrease of the flow and achieves the maximum with the lowest flow, i.e., 1.8 L / min. For comparison of studies on the non-insulated and insulated receiver the efficiency graph was made (figure 14).

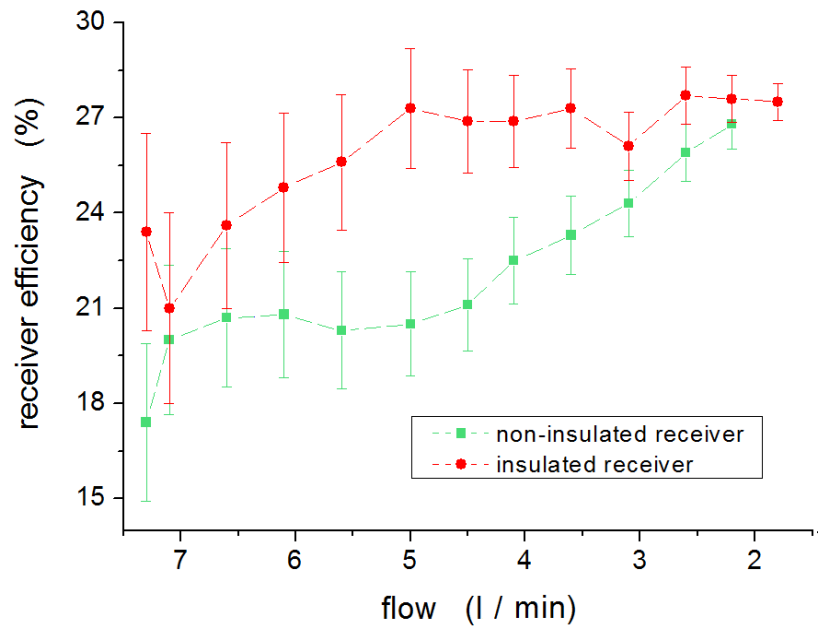

Figure 14. The efficiency of heat receiver in varied flow.

It has been assumed in the calculations that the concentrator system has the efficiency of $100 \%$, i.e. the enhancement of light on the exchanger equals 85 , what was calculated from the ratio of the concentrator surface to the receiver surface. It was assumed that the whole solar radiation falling on the concentrator bowl surface is reflected, and all rays reach the absorber surface. In reality, taking into account the efficiency of the concentrating system will contribute to the increase of the calculated values of the receiver efficiency.

In the graph in figure 14 it can be seen that the thermal insulation of mineral wool contributed to the increase of efficiency of the receiver system. Effectiveness in both cases is higher for lower flows, what is caused by achieving a greater difference of temperatures before and behind the receiver (figure 12). At the flow of $5 \mathrm{~L} / \mathrm{min}$ it is visible that the use of insulation significantly increases the efficiency of heat reception. The studies did not include the impact of wind during the measurement, what also has the effect on the presented results. However, it can be noticed that the best results are obtained for the lowest flows.

Generally the efficiency of non-insulated receiver was estimated at the level of $22.9 \pm 0.5 \%$, however insulated receiver reached the value of $26.6 \pm 0.3 \%$. So even small thickness of insulation material led to important improvement of its efficiency.

\section{Conclusions}

The study results presented in this article are the basis for the continuation of research into the possibility of obtaining the high-temperature heat from the solar system for producing cold. An important element of the discussed system is the receiver of the high-temperature heat. Initially, the efficiency of the system was increased by using the insulation of mineral wool, what in average raised the effectiveness more than $15 \%$.

To raise the efficiency of the absorption system, conversion and heat reception it is necessary to introduce next modifications. Currently, the works are ongoing about, among others, the application of the absorption surface with the lowest possible emissivity. Also the external shape of the receiver is very important. The absorption surface should be constructed in such a way so that the concentrated rays fall on it at the straight angle, what will contribute to increasing the absorption efficiency. Extremely important here is also the optimal placement of the receiver in the concentrator system. In studies on increasing the radiation absorption efficiency the simulation tools are useful, which use the "ray tracing" technique [25]. Also the efficient reception of heat is important, thus the internal geometry of the receiver. The simulation of the medium flow and different work parameters of the system are conducted at the same time to experimental works using the ANSYS software based on CFD methods.

The examples of the solar systems used in the World for producing cold quoted in the article and the presented results confirm that using only the solar power without the help of the additional heat source can be an effective way for producing cold in the summer period. The biggest advantage of this type of systems is the fact that in case of their application the coherence occurs, that the largest heat production occurs in the summer period, i.e., when the greatest demand for cold takes place. 


\section{Acknowledgments}

This work was financially supported by Dean Grant of Faculty of Energy and Fuels at the AGH University of Science and Technology and in was also completed as a part of the statutory activities of the Department of Sustainable Energy Development "Studies concerning the conditions of sustainable energy development".

\section{References}

1. Directive $2009 / 28 / \mathrm{EC}$

2. Directive $2010 / 31 / \mathrm{EU}$

3. Directive $2008 / 50 /$ EC

4. P. McKeen, A. S. Fung, Buildings, 4, 336-354 (2014)

5. A. Q. Jakhrani, S. R. Samo, A. R. H. Rigit, S. A. Kamboh, WASJ 22 (9), 1334-1343 (2013)

6. Europe's Energy Portal: https://www.energy.eu/

7. P. Shi, C. Jaeger, Q. Ye, Integrated Risk Governance, IHDP, 218 (2013)

8. B. Gradoń, S. Gil, W. Bialik, Polska Energetyka Słoneczna, 1-4, 55-59 (2012)

9. R. E. Critoph, Polska Energetyka Słoneczna, 1-4, 14-19 (2009)

10. A.B. Jemaa, S. Rafa, N. Essounbouli, A. Hamzaoui, F. Hnaien, F. Yalaoui, Energy Procedia, 42, 406415 (2013)

11. J. Stefaniak, Rocznik Ochrona Środowiska, 15, 1216-1227 (2013)

12. B.H. Gebreslassie, G. Guillen-Gosalbez, L. Jimenez, D. Boer, Energy, 35, 3849-3862 (2010)

13. M. Turski, R. Sekret, Architecture Civil Engineering Environment, 2, 109-115 (2012)

14. M. Turski, R. Sekret, Instal, 9, 47-50 (2013)

15. M.S. Fernandes, G.J.V.N. Brites, J.J. Costa, A.R. Gaspar, V.A.F. Costa, Renewable and Sustainable Energy Reviews, 39, 102-123 (2014)

16. E.E. Anyanwu, Energy Conversion and Management, 44, 2, 301-312 (2003)

17. A.H.H. Ali, P. Noeres, P. Pollerberg, Solar Energy, 82, 1021-1030 (2008)

18. A. Syed, M. Izquierdo, P. Rodriguez, G. Maidment, J. Missenden, A. Lecuona, R. Tozer, International Journal of Refrigeration, 6/28, 859-871 (2005)

19. Technical specification of WFC-SC5 YAZAKI absorption chiller

20. P. Bermejo, F. J. Pino, F. Rosa, Solar Energy, 84, 1503-1512 (2010)

21. M. Balghouthi, M.H. Chahbani, A. Guizani, Applied Energy, 98, 138-148 (2012)

22. I. Sarbu, C. Sebarchievici, Energy Conversion and Management, 105, 403-422 (2015)

23. K.R. Ullah, R. Saidur, H.W. Ping, R.K. Akikur, N.H. Shuvo, Renewable and Sustainable Energy Reviews, 24, 499-513 (2013)

24. I. Sarbu, C. Sebarchievici, Energy and Buildings, 67, 286-297

25. E. Bożek, M. Filipowicz, JCEEA, 26, 2, 19-28 (2015) 